\title{
High-energy non-resonant X-ray magnetic scattering from $\mathrm{EuAs}_{3}$
}

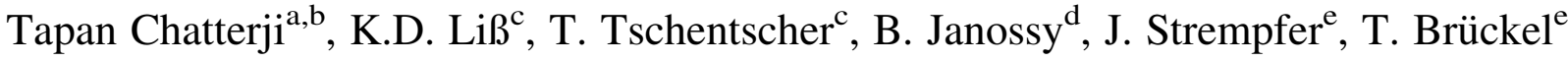 \\ anstitut Laue-Langevin, BP 156, 38042 Grenoble Cedex 9, France \\ ${ }^{\mathrm{b}}$ Max-Planck-Institut für Physik Komplexer Systeme, Nöthnizerstrasse 32, D-01187 Dresden, Germany \\ ${ }^{\mathrm{c}}$ European Synchrotron Radiation Facility, BP 220, F-38043 Grenoble Cedex, France \\ ${ }^{\mathrm{d}}$ Ecole Polytechnique Fédérale de Lausanne, CH-1015 Lausanne, Switzerland \\ ${ }^{\mathrm{e}}$ HASYLAB, DESY, Notkestrasse 85, D-22603 Hamburg, Germany
}

Received 30 April 2004; accepted 14 June 2004 by C. Lacroix

Available online 14 July 2004

\begin{abstract}
We have investigated non-resonant high energy X-ray magnetic scattering from $\mathrm{EuAs}_{3}$ both in the antiferromagnetic and in the incommensurate phase by using an X-ray energy of 104 and $106 \mathrm{keV}$. In the antiferromagnetic phase, we obtained a signal to background ratio of about 10:1 for the magnetic Bragg peak at $Q=(-1,0,1 / 2)$ and a maximum count rate of about 200 counts/s at $T=3.1 \mathrm{~K}$. To our knowledge this is the first reported observation of the non-resonant magnetic signal from a rareearth ion at X-ray energy as high as $106 \mathrm{keV}$. The temperature dependence of the integrated intensity of the $(-1,0,1 / 2)$; magnetic reflection has been measured and compared with that obtained previously by neutron diffraction. We measured the integrated intensities of several magnetic reflections from the antiferromagnetic phase and have compared them with those calculated from the magnetic structure model derived from neutron diffraction. The intensities of the magnetic satellite reflections from the incommensurate phase have been measured and have been found to be very weak. We also investigated the temperature variation of the lattice spacing close to the magnetic ordering transition and have found a large magnetoelastic anomaly at the lock-in phase transition.
\end{abstract}

(C) 2004 Elsevier Ltd. All rights reserved.

PACS: 75.25

Keywords: A. Rare earth compounds; C. High energy X-ray magnetic scattering; C. Incommensurate structures; D. Lock-in phase transition; D. Magnetoelastic effect

The use of high energy ( $\sim 100 \mathrm{keV})$ non-resonant X-ray magnetic scattering in investigating the model antiferromagnetic material $\mathrm{MnF}_{2}$ with $3 \mathrm{~d}$ ions has been shown recently $[1,2]$. This technique has several important advantages over the conventional resonant $\mathrm{X}$-ray magnetic scattering technique: (1) it probes the bulk of the material, (2) extinction free measurement of the magnetic intensities

\footnotetext{
* Corresponding author. Science Department, Institut LaueLangevin, BP 156, 38042 Grenoble Cedex 9, France. Tel.: +330476-207066; fax: + 33-04862-07648.

E-mail address: chatt@ill.fr (T. Chatterji).
}

of several magnetic reflections is possible, (3) the measured magnetic intensities with low $Q$ can yield the magnetic spin moments $S$ of the scattering ions, (4) high energy X-rays can penetrate sample environment vessels easily. Combining with neutron scattering technique which measures the sum $2 S+L$ of spin and orbital angular momentum, a separation of spin and angular momentum is possible. The high energy $\mathrm{X}$-ray magnetic scattering cross-section has little or no polarization dependence. The Q-resolution obtained with a three-crystal diffractometer is about one order of magnitude higher than that obtained with high-resolution neutron diffraction. Additionally owing to the large penetration depth of the high energy $\mathrm{X}$-rays, an enhancement of the cross-section 
of several orders of magnitude can be obtained for transition elements. However, the penetration depth is smaller for rareearth and actinides and therefore the volume enhancement is not as favorable as in the case of transition elements. Nevertheless here we show the potential use of this technique for investigating the magnetic properties of rare earth compounds.

$\mathrm{EuAS}_{3}$ is an example of a semimetallic system in which $\mathrm{p}-\mathrm{f}$ hybridization effects give rise to unusual magnetic properties. This compound orders at $T_{\mathrm{N}} \approx 11 \mathrm{~K}$ to an incommensurate sine wave phase which undergoes an incommensurate-commensurate lock-in phase transition at $T_{\mathrm{L}} \approx 10 \mathrm{~K}[3,4]$. The magnetic $(\mathrm{H}-\mathrm{T})$ and $(\mathrm{P}-\mathrm{T})$ phase diagrams are very complex containing several helimagnetic phases $[5,6]$. We have chosen $\mathrm{EuAs}_{3}$ as a model system because its magnetic properties have been thoroughly investigated by neutron scattering [3-6].

We have investigated non-resonant high energy X-ray magnetic scattering from $\mathrm{EuAs}_{3}$ both in the antiferromagnetic and the incommensurate phase by using X-ray energy of $104 \mathrm{keV}$ at the high energy beam line ID15 of the European Synchrotron Radiation Facility in Grenoble. Some preliminary measurements were performed on the high energy beam line BW5 of the HASYLAB, DESY in Hamburg using an X-ray energy of $106 \mathrm{keV}$. A single crystal of size $5 \times 1 \times 0.7 \mathrm{~mm}^{3}$ with a mosaic spread of about $0.02^{\circ}$ was used for the present investigation. The monoclinic $b$ axis of the crystal was fixed parallel to the $\omega$ axis of the diffractometer. The magnetic moments of the $\mathrm{Eu}$ ions are parallel to the monoclinic $b$ axis both in the commensurate and also in the incommensurate phase. We have performed the experiment in the three-crystal mode using oxygen precipitated $\mathrm{Si}$ (111) monochromator and analyzer crystals with mosaic spreads of about 5 arc seconds. The use of high energy X-rays enabled us to use a conventional helium cryostat with an aluminum tail without the use of any special windows.

A search for magnetic reflections at low temperatures readily revealed magnetic intensities in the antiferromagnetic phase corresponding to the propagation vector $\mathbf{k}=(-1,0,1 / 2)$. On the ID15 beam line of the ESRF we could get a signal to background ratio of about 10:1 and a maximum count rate of about 200 counts/s. Fig. 1 shows the sample rotation or $\omega$ scan and also analyzer rotation scan of the 1,0,5/2 magnetic reflection at several temperatures determined on the ID15 of the ESRF. Fig. 2 shows the temperature dependence of the integrated intensity of the 1,0,5/2 magnetic Bragg peak which decreases with increasing temperature. The intensity of this reflection drops abruptly to zero at the commensurate to incommensurate lock-in phase transition temperature $T_{\mathrm{L}} \approx 10 \mathrm{~K}$. We have normalized the intensity of the magnetic reflection by the machine current which decreases continuously with time after the injection. We succeeded in measuring the magnetic satellite Bragg peaks from the incommensurate amplitude modulated phase, which are rather weak. Fig. 3 shows the sample rotation scan of the 2.95,0,1.38 magnetic reflection of incommensurate magnetic phase at $T=10.2 \mathrm{~K}$. We have measured the intensities of several magnetic reflections from the antiferromagnetic phase. In order to put the magnetic intensities in absolute scale we have also measured the intensities of the corresponding charge reflections at the double $\mathrm{Q}$ positions along the radial direction. The magnetic structure factors have been compared with those calculated from the magnetic structure model derived from neutron diffraction investigations [3,4]. The observed and the calculated structure factors agree well within the experimental accuracy.

We have investigated the thermal expansion anomaly due to the magnetoelastic effects at the magnetic phase

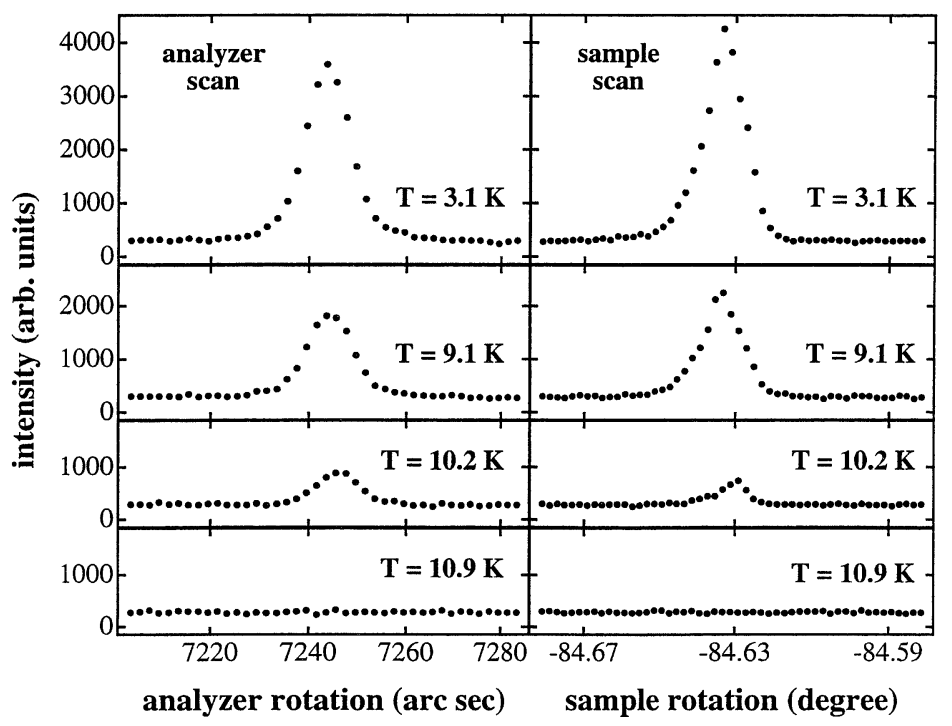

Fig. 1. Typical sample rotation or $\omega$ and the analyzer rotation scans of the 1,0,5/2 magnetic reflection of EuAs ${ }_{3}$ at several temperatures. 


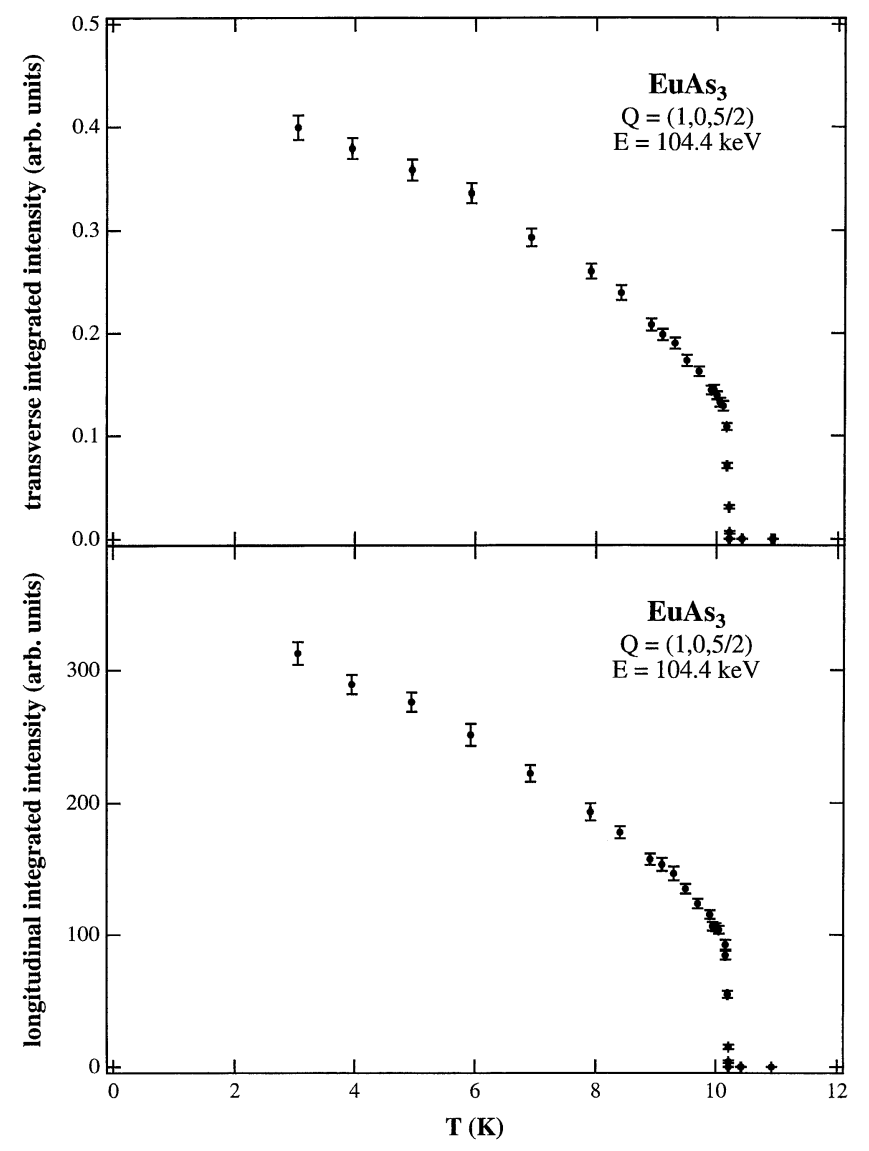

Fig. 2. Temperature dependence of the integrated intensity of the 1,0,5/2 magnetic reflection determined by longitudinal (radial reciprocal direction) and transverse scans of $\mathrm{EuAs}_{3}$. The intensity decreases with increasing temperature and drops abruptly at the first-order commensurate-to in-commensurate lock-in transition at $T_{\mathrm{L}} \approx 10.2 \mathrm{~K}$. The results are very similar to those obtained previously [3,4] by neutron diffraction. We have detected the satellite reflections corresponding to the incommensurate phase (see Fig. 3) but did not determine its temperature variation due to their very small intensities. The temperature variation of the satellite intensities has been thoroughly investigated both by neutron $[3,4]$ and resonant X-ray diffraction at the Eu L absorption edge [8].

transition. We have determined the temperature variation of the angular position of the $-6,0,6$ charge reflection as a function of temperature. For this measurement we used perfect Si (351) crystals as the monochromator and the analyzer crystals. Ideally the $Q=2 \pi / d=4 \pi \sin \theta / \lambda$ values of the scattering planes of the monochromator, the sample and the analyzer crystals should be equal for the full width at the half maximum (FWHM) of the analyzer scan to be independent of the mosaic spread of the sample. The $Q$ of the $\mathrm{Si}$ (351) is $6.84435 \AA^{-1}$ whereas the $Q$ of the $-6,0,6$ of $\mathrm{EuAs}_{3}$ is about $6.75 \AA^{-1}$ Since the angular position of this reflection changes by a few arc seconds only at the phase transition we employed an interferometric method to monitor the angles and measure them [7]. The monochromator, the sample and the analyzer had coarse rotation stages for alignment and setup, and piezodriven fine rotation stages. The readout of the actual settings was done by optical interferometers. The precision of the angular setting was about 0.1 arc second. Experimentally the FWHM of the

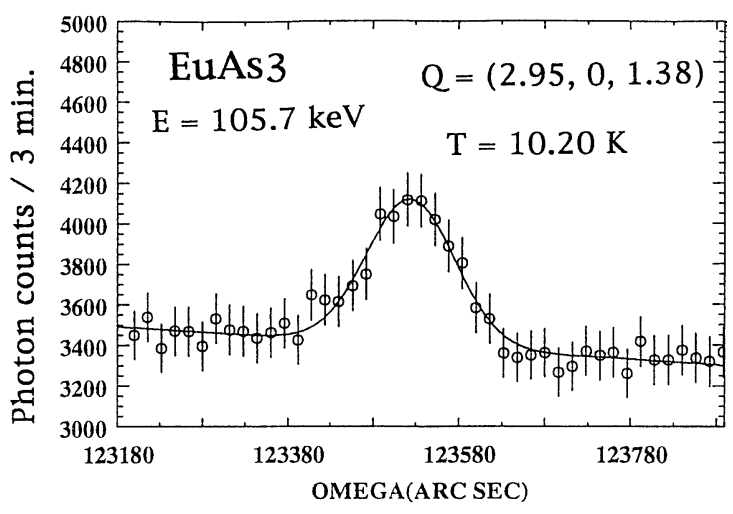

Fig. 3. Sample rotation or $\omega$ scan of the 2.95,0,138 magnetic reflection of the incommensurate magnetic phase of $\mathrm{EuAs}_{3}$ at $T=$ $10.2 \mathrm{~K}$. 

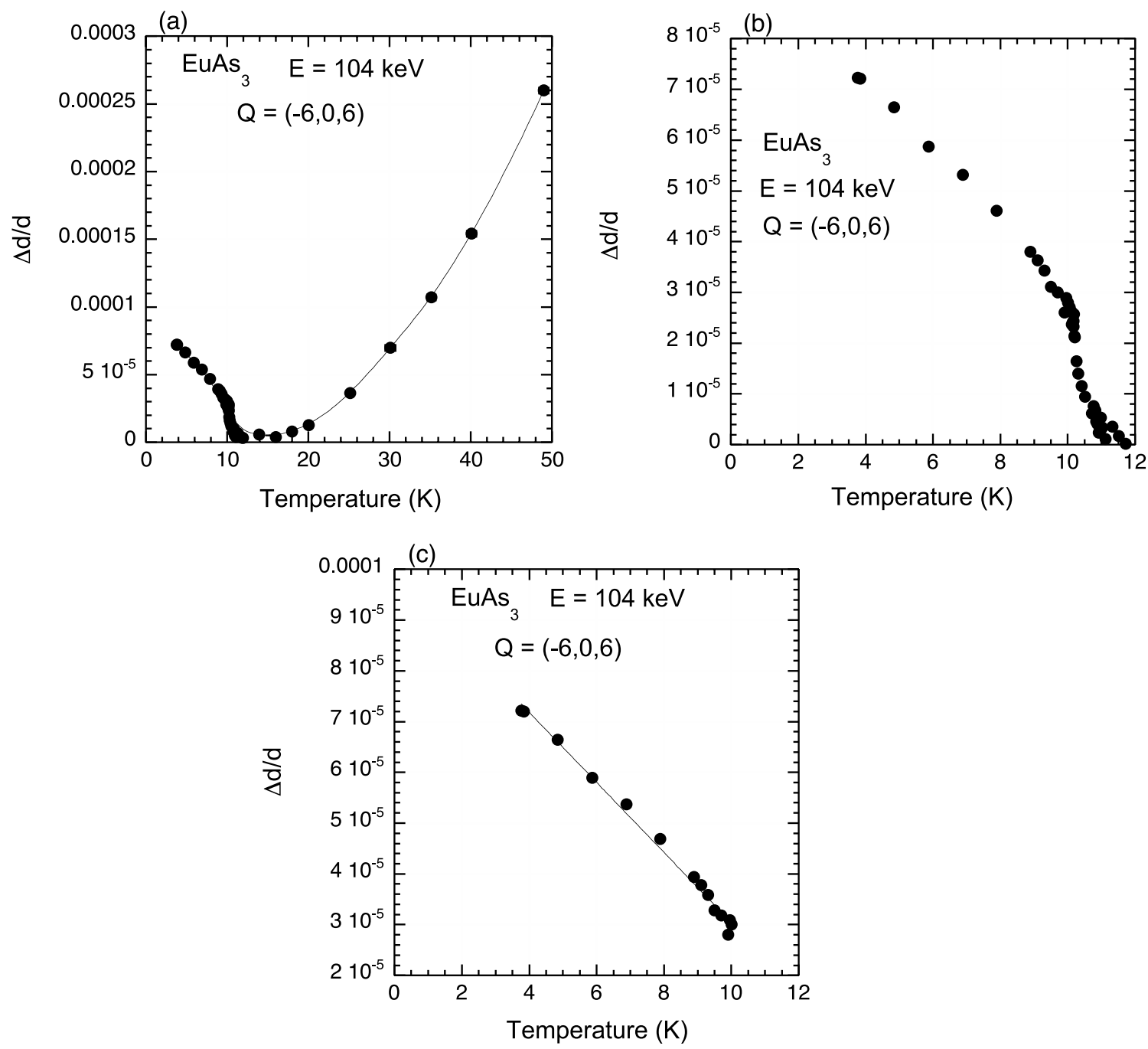

Fig. 4. (a) Temperature variation of the relative change in lattice spacing $\Delta d / d$ corresponding to the $-6,0,6$ charge reflection. The continuous curve is a guide to the eye. (b) Temperature variation of the relative lattice spacing $\Delta d / d$ close to the magnetic transitions, $T_{\mathrm{L}} \approx 10.3 \mathrm{~K}$ and $T_{\mathrm{N}} \approx 11 \mathrm{~K} . \Delta d / d$ decreases linearly with increasing temperature up to about the lock-in temperature $T_{\mathrm{L}} \approx 10 \mathrm{~K}$. $\Delta d / d$ decreases more rapidly in the temperature range $10 \mathrm{~K} \leq T \leq 11 \mathrm{~K}$ in the incommensurate phase. (c) Temperature variation of $\Delta d / d$ in the commensurate antiferromagnetic phase. The continuous curve is the linear fit of the data.

analyzer scan of the -606 reflection was about 1.6 arc second. Fig. 4 shows the temperature variation of the relative change in lattice spacing $\Delta d / d$. For systems without any magnetic phase transition one expects the $\Delta d / d$ value to decrease with decreasing temperature and finally attain a constant value at very low temperatures. In the present case the $\Delta d / d$ value decreases with decreasing temperature and becomes almost zero at about $T=12 \mathrm{~K}$, but starts increasing at about $T_{\mathrm{N}} \approx 11 \mathrm{~K}$ with decreasing temperature. The $\Delta d / d$ value increases abruptly below the lock-in phase transition $T_{\mathrm{L}} \approx 10 \mathrm{~K}$. At lower temperature $\Delta d / d$ is found to increase linearly with decreasing temperature. We interpret this increase in the $\Delta d / d$ value at low temperatures to be due to the magnetoelastic effects at the magnetic phase transitions.
We have measured this effect only along $(-1,0,1)$ which is in the monoclinic $\mathrm{a}-\mathrm{b}$ plane.

The present results demonstrate that the high energy nonresonant X-ray magnetic scattering is a useful complimentary technique for investigating magnetic properties of materials. Unlike the medium energy resonance $\mathrm{X}$-ray magnetic scattering, the high-energy non-resonant X-ray magnetic scattering probes the bulk material and magnetic structure factors determined from this technique yield spin moments of the magnetic atoms. So this technique when combined with neutron diffraction which measures the total $2 S+L$ moment, can in principle yield individual values of $S$ and $L$. We have also demonstrated that due to superior Q resolution the magnetoelastic effects accompanying 
magnetic phase transition can be conveniently investigated by this technique.

\section{References}

[1] M. Lippert, T. Brückel, T. Köhler, J.R. Schneider, Europhys. Lett. 27 (1994) 537.

[2] J. Strempfer, T. Brückel, U. Rütt, J.R. Schneider, K.-D. Liss, T. Tchentscher, Acta Crystallogr. A52 (1996) 438.
[3] T. Chattopadhyay, P.J. Brown, P. Thalmeier, H.G. von Schnering, Phys. Rev. Lett. 57 (1986) 372; T. Chattopadhyay, P.J. Brown, H.G. von Schnering, Europhys. Lett. 6 (1988) 89.

[4] T. Chattopadhyay, P.J. Brown, P. Thalmeier, W. Bauhofer, H.G. von Schnering, Phys. Rev. B 37 (1988) 269.

[5] T. Chattopadhyay, P.J. Brown, Phys. Rev. B 38 (1988) 795.

[6] T. Chattopadhyay, P.J. Brown, Phys. Rev. B 41 (1990) 4358.

[7] P. Suortti, T. Tschentscher, Rev. Sci. Instrum. 66 (1995) 1798.

[8] T. Chattopadhyay, K.D. Liss, T. Brückel, J. Magn. Magn. Mater. 177-181 (1998) 1058. 